\title{
A METHOD OF WATER DEPTH INVERSION IN COASTAL AREA CONSIDERING TEMPERATURE INFORMATION
}

\author{
Yu Liu ${ }^{1}$, Xinyuan $\mathrm{Gao}^{1}$, Guanghui Wang ${ }^{1,2, *}$, Tao Zhang ${ }^{1}$, Jie Wang ${ }^{1}$ \\ ${ }^{1}$ Land Satellite Remote Sensing Application Center, MNR, Beijing 10048, China \\ ${ }^{2}$ School of Environment and Spatial Informatics, China University of Mining and Technology, Xuzhou 221116, China
}

Commission III, WG III/1

KEY WORDS: Water Depth, BP Neural Network, Sea Surface Temperature

\begin{abstract}
:
The remote sensing method for water depth inversion is fast, flexible, and low in cost, which has become an important means of method for water depth detection. This paper takes the coastal area where is around Gulangyu Island as the research area. Based on the spectral reflectance, sea surface temperature (SST) and measured water depth data, a nonlinear inversion model of water depth is established by using BP neural network. Combined with the tide data, the water depth and underwater topography in coastal area is obtained. The average relative error is 0.27 . The root mean square error is 1.92 . The results show that the participation of sea surface temperature in the model construction can improve the inversion error of offshore water depth to a certain extent, and can help improve the accuracy of the model.
\end{abstract}

\section{INTRODUCTION}

Shallow water depth is an important element of underwater topography. It is helpful to better understand the topography of the coastal area and develop the marine resources by measuring the water depth. echo sounding sounder is used in traditional water depth measurement. echo sounding sounder has the characteristics of high measurement accuracy and full coverage. However, this method is constrained by its inefficiency in shallow waters and very high operating logistic costs (Gao J, 2009; Jawak S D, et al.,2015). In comparison, Satellite remote sensing has becoming a efficient and cost-effective supplement to traditional measurements of water depth because of its high spatial, temporal and spectral resolution, as well as its advantages of immediacy, dynamic, accuracy and comprehensiveness (Su, et al.,2015), especially in remote and broad areas

The commonly used multispectral remote sensing water depth inversion methods can be divided into three categories: theoretical model, semi-empirical model and statistical model, such as linear regression methods, band ratio method, neural network method, etc. (Lyzenga D R, 1978; Stumpf R P, et al.,2003; Ceyhun Ö , et al, 2010; Bramante J F, et al., 2013). According to the theory of radiative transfer in the sea, the vertical water temperature has a certain relationship with water depth especially in the coastal area because of the underwater bottom topography. So, it is feasible to use thermal infrared image data to inverse water depth. Neural network has the ability to solve complex nonlinear mapping, and at the same time, it does not need to consider external water conditions during the water depth inversion process, which has greater advantages. In this paper, landsat 8 OLI/TIRS and Electronic Chart Display and Information System (ECDIS) data are used as data sources to build the model. Based on BP neural network, the mapping relationship between sea surface temperature, reflectance and water depth is established to realize the inversion of water depth in coastal area. Furthermore, we can explore the feasibility of temperature assisted extraction of water depth.

\section{STUDY AREA AND DATA}

\subsection{Overview}

The study area is located around Gulangyu Island in the Western sea area of Xiamen, China, as shown in Figure 1. The average sea water temperature is $21^{\circ} \mathrm{C}$, and the annual sea water temperature ranges from $13{ }^{\circ} \mathrm{C}$ to $33{ }^{\circ} \mathrm{C}$. The tide type in the sea area is a regular semi-diurnal tide, with an average tidal range of 3.98 meters. The study area has a high degree of development and frequent human activities.

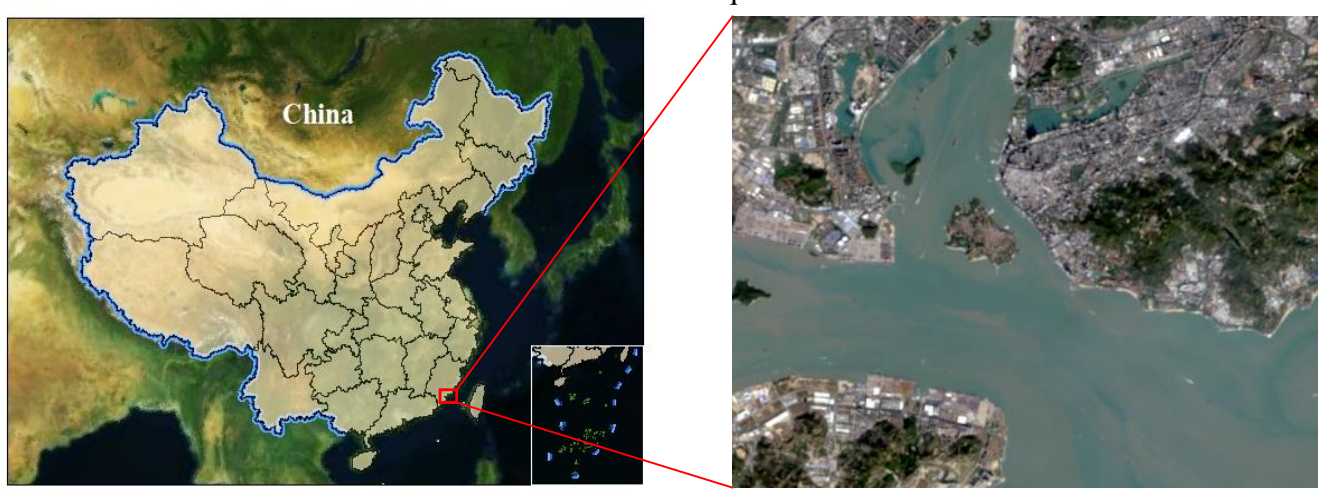

Figure 1. Study area

\footnotetext{
* Corresponding author
} 


\subsection{Data sources and pre-process}

In this paper, landsat8 OLI/TIRS is used to obtain sea surface reflectance and temperature. The sensor parameters are shown in Table1. The measured water depth data is obtained by vectorization of the ECDIS. Tide data is provided by China Oceanic Information network (http://globaltide.nmdis.org.cn/Default.html). In order to retrieve more accurate sea depths, reduce errors that caused by data, preprocesses like radiometric calibration, atmospheric correction, ortho-rectification has been done. Although the sea surface is calm at the time of imaging, there are still a small amount of noise such as solar flare and white cap caused by wave breaking, so wavelet transform is used for denoising. In order to retrieve the water depth, the water area is extracted and the image is cropped.

\begin{tabular}{llrc}
\hline \multicolumn{2}{c}{ Band } & $\begin{array}{c}\text { Resolution } \\
(\mathbf{m})\end{array}$ & $\begin{array}{c}\text { Spectral range } \\
(\boldsymbol{\mu} \mathbf{m})\end{array}$ \\
\hline Band1 & Coastal/ & 30 & $0.435-0.451$ \\
& Aerosol & 30 & $0.452-0.512$ \\
Band2 & Blue & 30 & $0.533-0.590$ \\
Band3 & Green & 30 & $0.636-0.673$ \\
Band4 & Red & 30 & $0.851-0.879$ \\
Band5 & NIR & 30 & $1.566-1.651$ \\
Band6 & SWIR-1 & 30 & $2.107-2.294$ \\
Band7 & SWIR-2 & 15 & $0.503-0.676$ \\
Band8 & Pan & 30 & $1.363-1.384$ \\
Band9 & Cirrus & 100 & $10.60-11.19$ \\
Band10 & TIR-1 & 100 & $11.50-12.51$ \\
Band110 & TIR-2 & 30 \\
\hline
\end{tabular}

Table 1. Main parameters for the Landsat8 OLI/TIRS

\section{RESEARCH METHOD}

\subsection{Sea surface temperature retrieval}

Make full use of the sensitivity of thermal infrared band to heat, the land surface temperature can be extracted (Liu,2018). In this paper, JM\&S single-channel algorithm proposed by JimënezMuñoz and Sobrino (2004) is used for sea surface temperature inversion. The calculation formula is as follows:

$$
\begin{gathered}
T s=\gamma\left[\frac{\psi_{1} L_{\text {sen }}+\psi_{2}}{\varepsilon}+\psi_{3}\right]+\delta \\
\gamma \approx \frac{T_{\text {sen }}^{2}}{b_{\gamma} L_{\text {sen }}} \\
\delta \approx T_{\text {sen }}-\frac{T_{\text {sen }}^{2}}{b_{\gamma}} \\
b_{\gamma}=c_{2}\left(\frac{\lambda^{4}}{c_{1}}+\frac{1}{\lambda}\right)
\end{gathered}
$$

Where $\varepsilon$ is emissivity, $\mathrm{L}_{\text {sen }}$ is radiance measured on the satellite $\left(\mathrm{w} \cdot \mathrm{m}^{-2} \bullet \mathrm{sr}^{-1} \mu \mathrm{m}^{-1}\right), \mathrm{T}_{\mathrm{sen}}$ is brightness temperature, $\lambda$ is the central wavelength, $c_{1}=1.91104 \times 10^{8} \mathrm{w} \cdot \mu \mathrm{m}^{4} \cdot \mathrm{m}^{-2} \cdot \mathrm{sr}^{-1}$, $c_{2}=14387.7 \mu \mathrm{m} \bullet \mathrm{k} . \quad \psi_{1}, \psi_{2}, \psi_{3}$ are solved by quadratic polynomials established with water vapor content. JimënezMuñoz et al. give specific parameter values for the landsat8 TIRS band10, the formula is as follows:

$$
\begin{gathered}
\psi_{1}=0.0419 \omega^{2}+0.02916 \omega+1.01523 \\
\psi_{2}=-0.38333 \omega^{2}-1.50294 \omega+0.2324
\end{gathered}
$$

$$
\psi_{3}=0.00918 \omega^{2}+1.3672 \omega-0.27514
$$

Where $\omega$ is water vapor content. $\omega$ can be obtained from MODIS. Related research shows that when the water vapor content $\omega$ is greater than $3 \mathrm{~g} \cdot \mathrm{m}^{-2}$, the accuracy of the algorithm will be significantly affected. Because the study area is located in the coastal area, the water vapor content is relatively large, so $\psi$ is calculated by the original formulas. the formula is as follows(Jiménez-Muñoz J C, et al., 2014):

$$
\psi_{1}=\frac{1}{\tau}, \psi_{2}=-L_{\downarrow}-\frac{L_{\uparrow}}{\tau}, \psi_{3}=L_{\downarrow}
$$

Where $\tau$ is Atmospheric transmittance, $L_{\downarrow}$ is atmospheric upward radiance, $L_{\downarrow}$ is atmospheric downward radiance. They can be obtained from NASA atmospheric correction parameter calculator website(https://atmcorr.gsfc.nasa.gov/).

\subsection{Water depth inversion model construction}

Remote sensing water depth inversion model is a nonlinear complex model, which is suitable to be approximated by artificial neural network (Liang Z C et al., 2012) . BP neural network simulates the working principle of the brain nerve tissue, and its structure is divided into input layer, hidden layer and output layer. There are full links between adjacent neurons in BP neural network, but no links between neurons in the same layer. The structure is shown in Figure2.

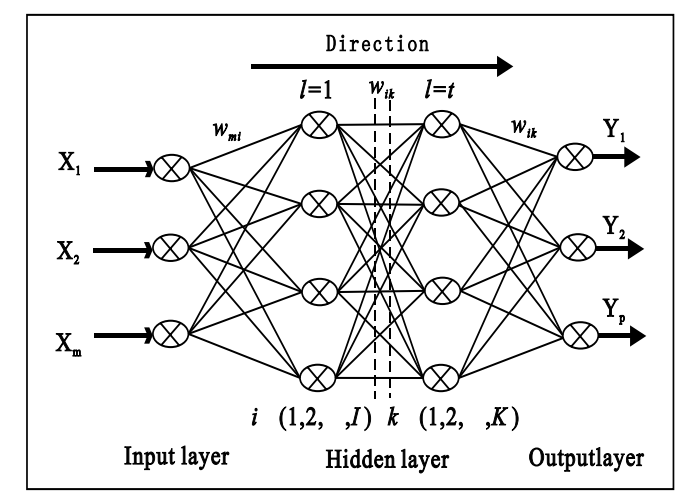

Figure 2. BP neural network structure

$$
Z=f(W \cdot X+B)
$$

Where $\mathrm{f}$ is the training function, $Z=\left[z_{1}, z_{2} \ldots z_{n}\right]^{T}$ is the vector of output layer, $W=\left[w_{1}, w_{2} \ldots w_{n}\right]^{T}$ is the weight matrix, $X=$ $\left[x_{1}, x_{2} \ldots x_{n}\right]^{T}$ is the vector of input layer, $B=\left[b_{1}, b_{2} \ldots b_{n}\right]^{T}$ is threshold.

In this paper, softmax regression algorithm is used to establish the BP neural network. The input data of BP neural network are coordinate information, spectral reflectance, sea surface temperature and water depth. Firstly, in order to speed up the network learning speed, the sample data is normalized. Then the connection weights and thresholds of BP neural network are generated randomly and the water depth is calculated. After obtaining the error between the inverted water depth and the true water depth, the gradient descent method is used to promote the convergence of the result.

In this paper, the activation function is sigmoid, the formula is as follows: 


$$
f=\frac{1-e^{-x}}{1+e^{-x}}
$$

Where $f$ is the output vector, $\mathrm{x}$ is the input vector. The error function is as follow:

$$
E=\frac{1}{2}(D-Y)^{T}(D-Y)
$$

Where $\mathrm{D}$ is the desired output values, $\mathrm{Y}$ are the actual output values. The partial derivative of each neuron in the output layer and the output of each neuron in the hidden layer are used to modify the weight $W$ by the error function, the formula is as follows:

$$
\Delta W_{1}=-\mu \frac{\partial E}{\partial W_{1}}
$$

Where $\mu$ is the learning rate. The partial derivative of each neuron in the hidden layer and the output of each neuron in the input layer are used to modify the weight $W$ by the error function, the formula is as follows:

$$
\Delta W_{2}=-\mu \frac{\partial E}{\partial W_{2}}
$$

When the error is less than the preset accuracy or the number of learning times is greater than the set number of training times, the calculation ends, otherwise the last round of learning is repeated until it meets the requirements.

\subsection{Tide correction}

With the change of time, the instantaneous tide level of sea water is constantly changing. The acquisition time of the measured water depth data cannot be consistent with the imaging time of the remote sensing data. The water depth inversion from the remote sensing image is different from the actual measured water depth. Therefore, after the water depth inversion, the tidal correction is needed. The actual water depth data can be calculated by adding the water depth data and the instantaneous tide height which can obtain from the China Oceanic Information network, as shown in Figure 3.

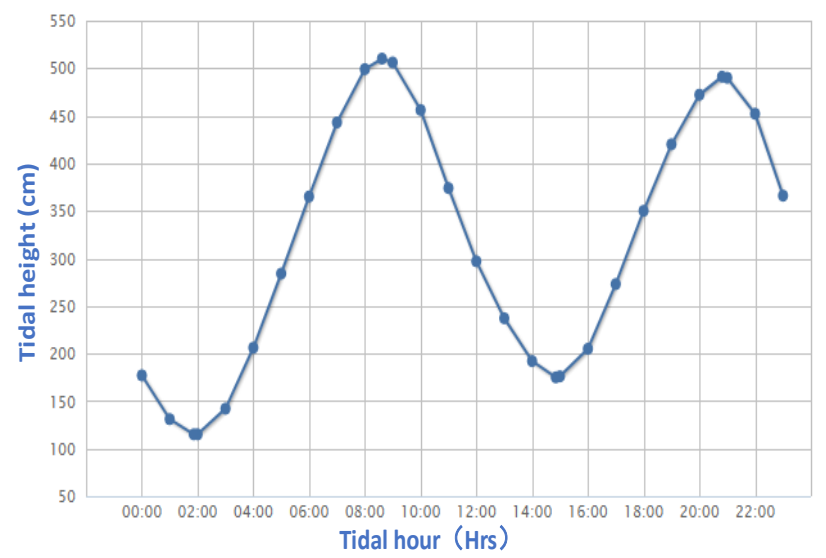

Figure3. Tide correction curve

\subsection{Accuracy evaluation}

In this paper, average relative error $(\sigma)$ and root mean square error $(\delta)$ are used to evaluate the accuracy of water depth inversion (Fan et al.,2015). The formula is as follows:

$$
\begin{gathered}
\sigma=\frac{\sum_{i=1}^{n}\left|\Delta z_{i} / z_{i}\right|}{n} \\
\delta=\sqrt{\frac{\sum_{i=1}^{n}\left(\Delta z_{i}\right)^{2}}{n}}
\end{gathered}
$$

Where $\Delta z_{i}$ is the difference between the measured water depth and the inversion water depth at the i-th check point. $z_{i}$ represents the measured water depth of the $\mathrm{i}$-th check point. $\mathrm{n}$ is the number of check points. The smaller the value of $\sigma$, the smaller the absolute value of the error between the measured water depth and the inversed water depth, which represents that the inversion result is reliable. The smaller the value of $\delta$, the smaller the fluctuation of the water depth inversion error, which represents that the inversion result is good.

\section{RESULT}

\subsection{Water depth inversion}

According to the ECDIS, 120 depth points are extracted, 80 of which are used for model building and 40 for accuracy verification. The distribution of sample points is shown in the figure4.

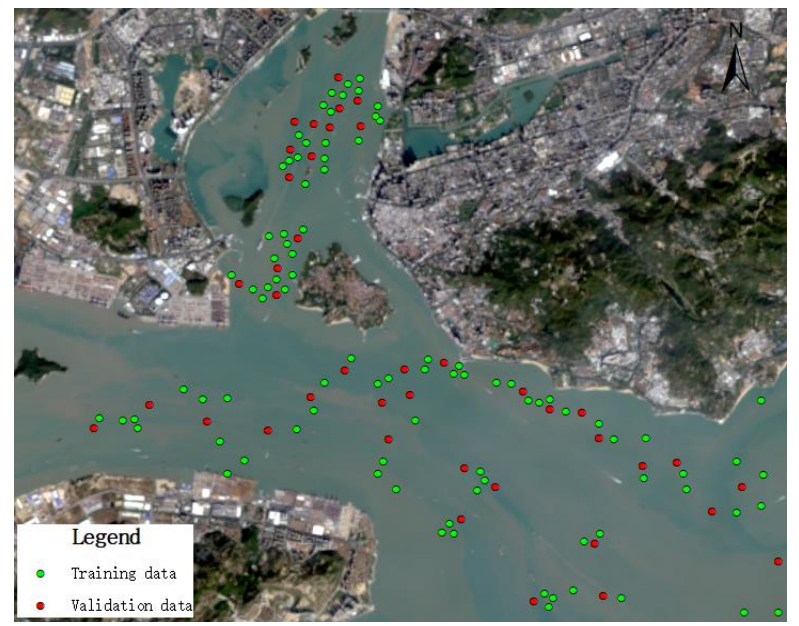

Figure4. Distribution of sample points

In clear water area, the effective penetration depth of blue band and green band can exceed $30 \mathrm{~m}$. In the turbid water area, the ability of detecting becomes weaker due to the large attenuation coefficient caused by the turbid water quality (Lafon V,2002 ). The reflectance of near infrared band is low, and it is sensitive to the water depth (Lyzenga D R,2006). The vertical water temperature has a certain relationship with water depth especially in the coastal area. So, the blue band, the green band, the near infrared band, the green band/the blue band and the sea surface temperature is selected as the water depth inversion factor. In order to keep the same resolution, the temperature inversion result is resampled to 30 meters.

BP neural network is constructed by the softmax function. The learning rate was 0.01 . The expected error is 0.1 . The number of iterations is 30000 . The number of hidden layer nodes is 15 . The average relative error of training accuracy is 0.11 . The root mean square error of training accuracy is 0.99 . Through this model, the inversion of water depth in the study area is realized. After the tide correction, the water depth inversion results are shown in the figure below. 

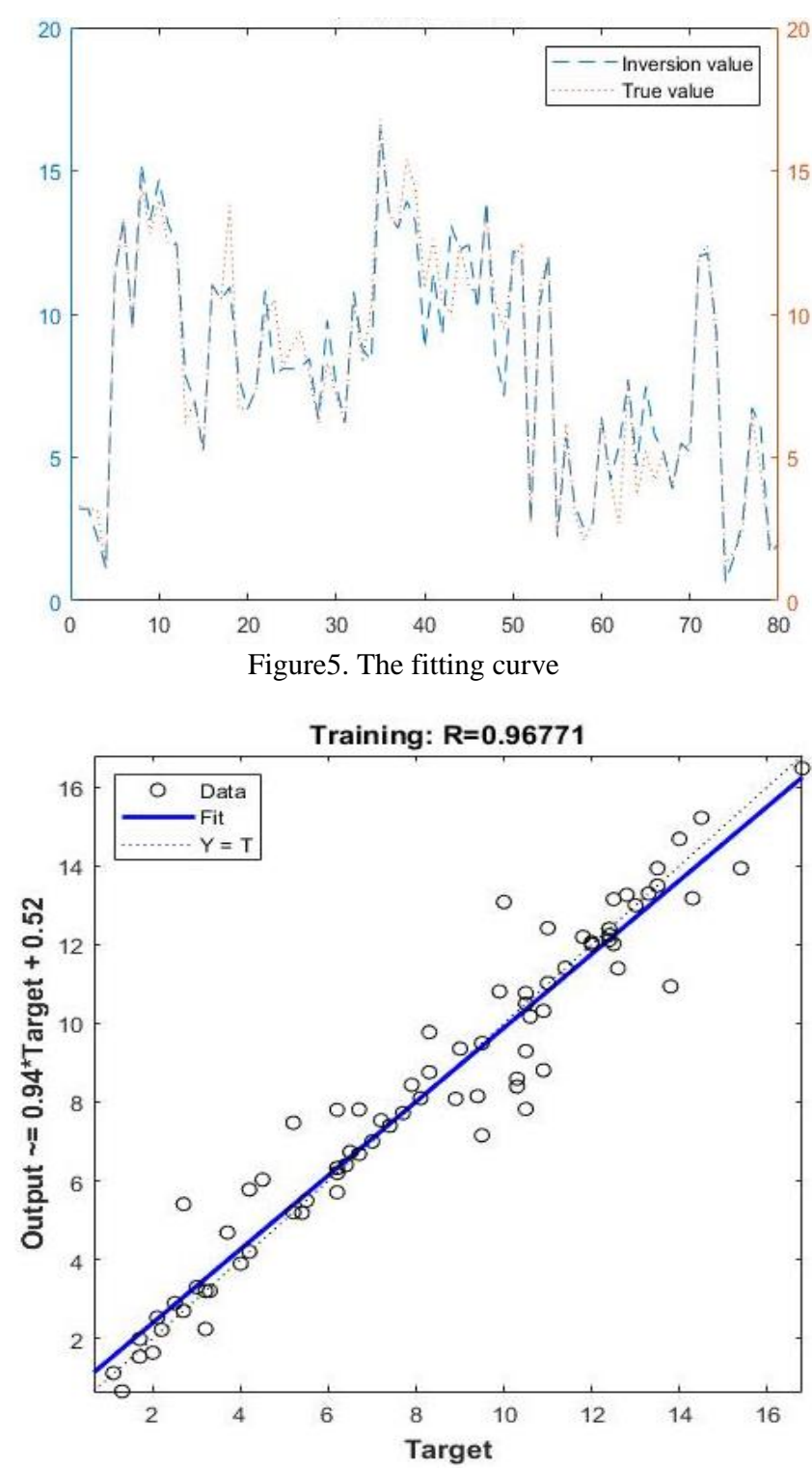

Figure6. The accuracy evaluation

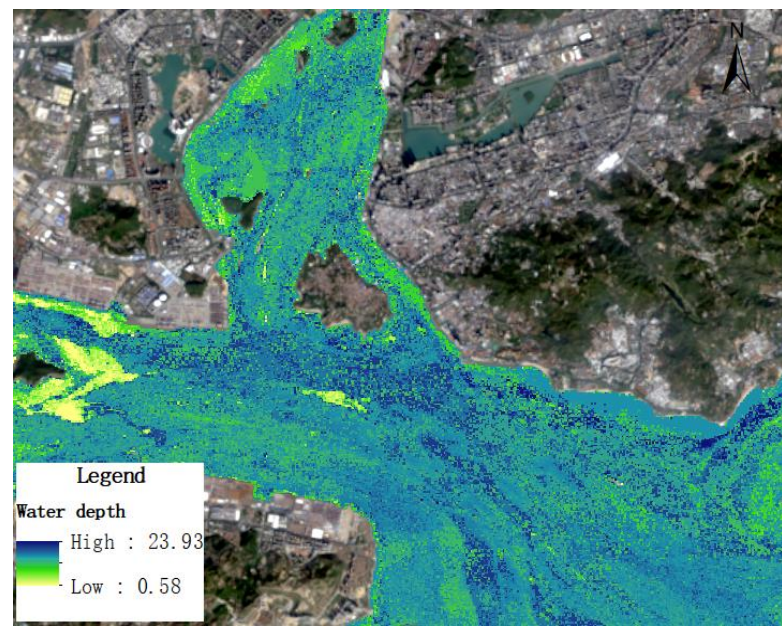

Figure7. The result of water depth inversion

\subsection{Accuracy evaluation and analysis}

The accuracy of the BP neural network model is verified by 40 check points. The average relative error is 0.27 . The root mean square error is 1.92 . Through visual interpretation, it can be found that the water depth inversion value in some areas is abnormal. According to the analysis, most of the abnormal points are near the land water boundary, and the water depth range is about 0 $7 \mathrm{~m}$. The reason is that the shallow sea area is greatly affected by natural factors such as sea breeze, chlorophyll concentration, suspended solid and others (Zhang,2020). The large attenuation coefficient of interference factors leads to the low accuracy of water depth inversion. With the increase of water depth, the accuracy is improved, and the effect of water depth inversion is better. In addition, the measured data of water depth come from the ECDIS, and there is a certain error between the measured data and the real water depth which will further increase the model error.

The initial weights and thresholds of BP neural network algorithm are generated randomly. In order to verify whether different initial weights and thresholds in training can lead to different results, two models are constructed in the same region using the same sample data and the same network structure, And use checkpoints to verify model accuracy. The results show that the average relative error of the two inversion results is 0.11 , which can be ignored for 30-meter resolution data. BP neural network model is not sensitive to initial weights and thresholds when the number of iterations is enough.

In order to judge whether the temperature information can assist the water depth inversion, the network structure with the same parameters is used in the case of no temperature information. The average relative error is 0.25 . The root mean square error is 2.01 . Taking the measured water depth value as the horizontal axis of the plane rectangular coordinate system and the inversion water depth value as the vertical axis. All the check points are drawn in the plane rectangular coordinate system, and the deviation between the inversion value and the measured value of the water depth inversion algorithm is obtained. For the convenience of expression, the straight line that passes through the origin of the coordinates and has a slope of 1 is called the "anastomosis line". The inversion value of all check points on the anastomosis line is equal to the actual measurement. The results are shown in Figure 8 and Figure 9. The average relative error of BP neural network model without temperature information is slightly higher, especially in shallow sea area.

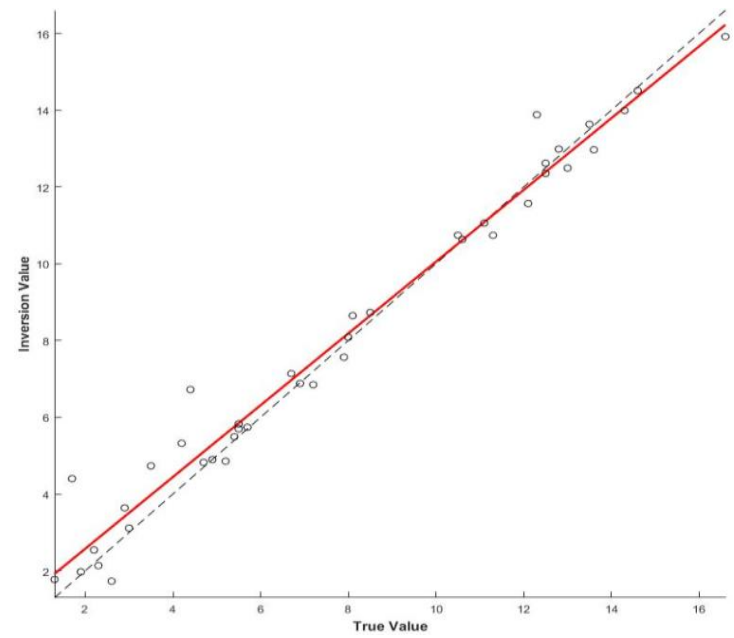

Figure8. Deviation chart 


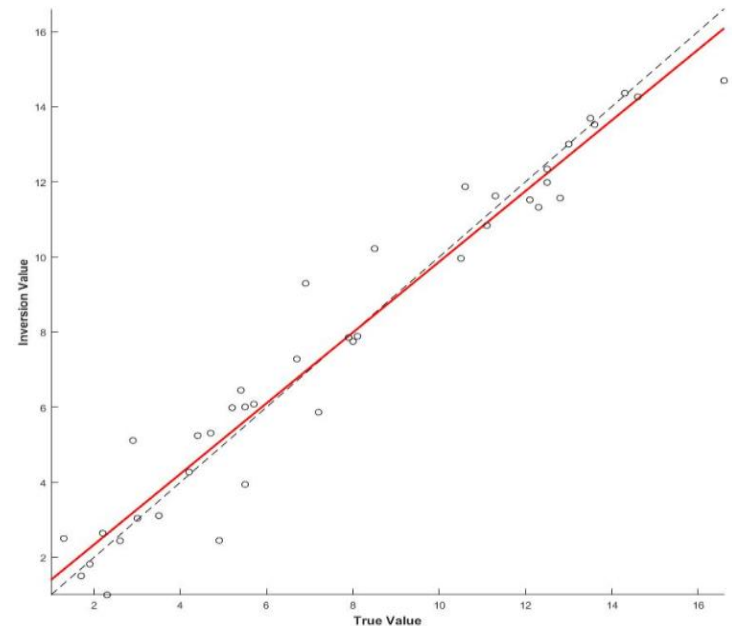

Figure9. Deviation chart without temperature

Water depth information reflects the topography of shallow seas. underwater topography in coastal area. Based on the water depth information, the visualization display of underwater topography can be realized as shown in Figure10.

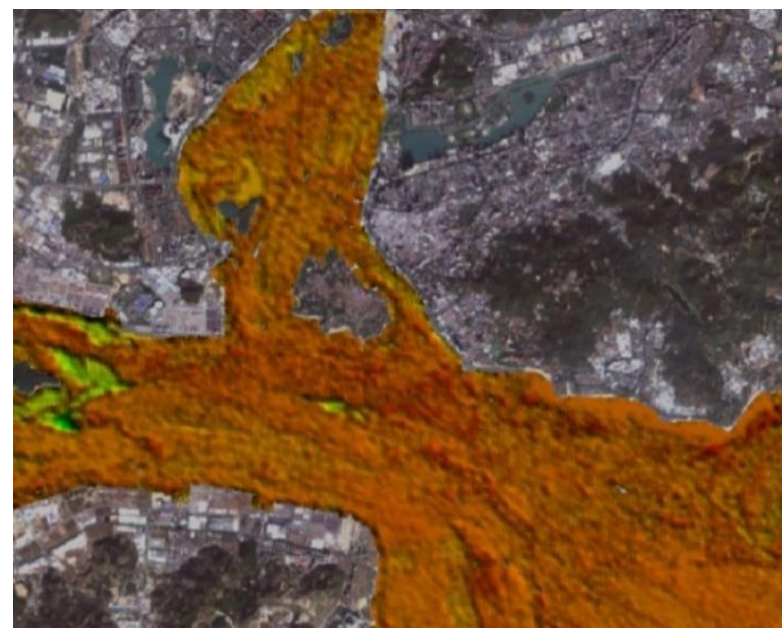

Figure10. Visualization display

\section{CONCLUSIONS}

In this paper, based on the BP neural network, the research of remote sensing water depth inversion is carried out. Combining the spectral reflectance and sea surface temperature, a water depth inversion model is constructed. Taking landsat8 OLI/TIRS as the data source, the test and analysis was complete. The main research conclusion received through the experiment is as follows:

1) The rapid inversion of water depth information is realized by using the BP neural network model. The average relative error is 0.27 . The root mean square error is 1.92 .

2) The participation of sea surface temperature in the model construction can improve the inversion error of offshore water depth to a certain extent, and can help improve the accuracy of the model.

3) The measured data of water depth comes from the ECDIS, so the timeliness and accuracy of the data can not be guaranteed. This will affect the accuracy of the water depth inversion model.
In the follow-up study, more and more accurate water depth points need to be obtained

\section{ACKNOWLEDGEMENTS}

This work was financially supported by the National Key Research and Development Program of China (no.2016YFB0501403) and the Major Program of China's HighResolutionEarth Observation System (NO. AH1601).

\section{REFERENCES}

Gao J, 2009. Bathymetric mapping by means of remote sensing: methods, accuracy and limitations. Progress in Physical Geography, 33(1), 103-116.

Jawak S D, Vadlamani S S, Luis A J, 2015. A synoptic review on deriving bathymetry information using remote sensing technologies: models, methods and comparisons. Advances in remote Sensing, 4(02), 147-162.

Su H, Liu H, Wu Q, 2015. Prediction of water depth from multispectral satellite imagery-the regression Kriging alternative. IEEE Geoscience and Remote Sensing Letters, 12(12), 2511-2515.

Lyzenga D R, 1978. Passive remote sensing techniques for mapping water depth and bottom features. Applied optics, 17(3), 379-383.

Stumpf R P, Holderied K, Sinclair M, 2003. Determination of water depth with high-resolution satellite imagery over variable bottom types. Limnology and Oceanography, 48(1part2),547556.

Ceyhun Ö, Yalçın A, 2010. Remote sensing of water depths in shallow waters via artificial neural networks. Estuarine, Coastal and Shelf Science, 89(1): 89-96.

Bramante J F, Raju D K, Sin T M, 2013. Multispectral derivation of bathymetry in Singapore's shallow, turbid waters. International journal of remote sensing,34(6), 2070-2088.

Sandidge J C, Holyer R J, 1998. Coastal bathymetry from hyperspectral observations of water radiance. Remote Sensing of Environment, 65(3), 341-352.

Lafon V, Froidefond J M, Lahet F, et al., 2002. SPOT shallow water bathymetry of a moderately turbid tidal inlet based on field measurements. Remote sensing of Environment, 81(1): 136-148.

Pacheco A, Horta J, Loureiro C, et al.,2015. Retrieval of nearshore bathymetry from Landsat 8 images: A tool for coastal monitoring in shallow waters. Remote Sensing of Environment, $159,102-116$

Lyzenga D R, Malinas N P, Tanis F J, 2006. Multispectral bathymetry using a simple physically based algorithm. IEEE Transactions on Geoscience and Remote Sensing, 44(8), 22512259.

Liceaga-Correa M A, Euan-Avila J I, 2002. Assessment of coral reef bathymetric mapping using visible Landsat Thematic Mapper data. International Journal of Remote Sensing, 23(1): 314. 
Liang Z C, Huang W Q, YANG Y, et al.,2012. Study of the water depth retrieval based on artificial neural network. Eng. Surveying Mapping, 21(4), 17-21.

Leu L G, Chang H W, 2005. Remotely sensing in detecting the water depths and bed load of shallow waters and their changes. Ocean Engineering, 32(10),1174-1198.

Liu Y, Liu Q, Fan W F, et al, 2018.Coupling Analysis of Heat Island Effects, Vegetation Coverage and Urban Flood in Wuhan. International Archives of the Photogrammetry, Remote Sensing and Spatial Information Sciences, XLII-3,1173-1177. doi.org/10.5194/isprs-archives-XLII-3-1173-2018.

Sobrino J A, Jiménez-Muñoz J C, Paolini L, 2004. Land surface temperature retrieval from LANDSAT TM 5. Remote Sensing of environment, 90(4), 434-440.

Jiménez-Muñoz J C, Sobrino J A, Skoković D, et al., 2014. Land surface temperature retrieval methods from Landsat- 8 thermal infrared sensor data. IEEE Geoscience and remote sensing letters, 11(10), 1840-1843.

Cao B, Qiu Z G, Cao B C, 2016. Comparison among Four Inverse Algorithms of Water Depth. Journal of Geomatics Science and Technology, 33(4),389-393

Fan Y G, Liu J X, 2015. Water depth remote sensing retrieval model based on artificial neural network techniques. Hydrographic Surveying and Charting, 35(4), 20-23.

Zhang X C, Ma Y, Zhang J Y, et al., 2020. Research on the remote sensing inversion fusion model of shallow water depth based on the piecewise adaptive algorithm. Marine Sciences, $44(6), 1-11$. 